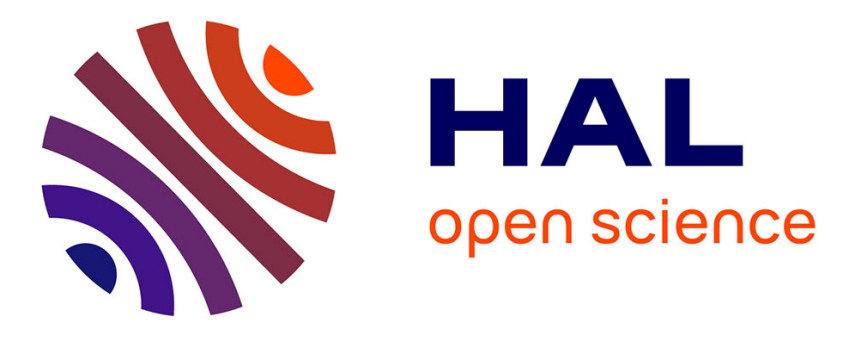

\title{
Making Gestural Interaction Accessible to Visually Impaired People
}

\author{
Anke Brock, Philippe Truillet, Bernard Oriola, Christophe Jouffrais
}

\section{To cite this version:}

Anke Brock, Philippe Truillet, Bernard Oriola, Christophe Jouffrais. Making Gestural Interaction Accessible to Visually Impaired People. 9th International Conference, EuroHaptics 2014, Versailles, France, June 24-26, 2014, Proceedings, Part II, Jun 2014, Versailles, France. http://link.springer.com/chapter/10.1007/978-3-662-44196-1_6, 10.1007/978-3-662-441961_6. hal-01078030

\section{HAL Id: hal-01078030 https://hal.science/hal-01078030}

Submitted on 27 Oct 2014

HAL is a multi-disciplinary open access archive for the deposit and dissemination of scientific research documents, whether they are published or not. The documents may come from teaching and research institutions in France or abroad, or from public or private research centers.
L'archive ouverte pluridisciplinaire HAL, est destinée au dépôt et à la diffusion de documents scientifiques de niveau recherche, publiés ou non, émanant des établissements d'enseignement et de recherche français ou étrangers, des laboratoires publics ou privés. 
This is the accepted author version. The final publication is available at http://link.springer.com/chapter/10.1007/978-3-662-44196-1_6

adfa, p. 1, 2014.

(C) Springer-Verlag Berlin Heidelberg 2014 


\title{
Making gestural interaction accessible to visually impaired people
}

\author{
Anke Brock, Philippe Truillet, Bernard Oriola, Christophe Jouffrais \\ IRIT-UMR5505, CNRS \& Université de Toulouse, France \\ Firstname.lastnamedirit.fr
}

\begin{abstract}
As touch screens become widely spread, making them more accessible to visually impaired people is an important task. Touch displays possess a poor accessibility for visually impaired people. One possibility to make them more accessible without sight is through gestural interaction. Yet, there are still few studies on using gestural interaction for visually impaired people. In this paper we present a comprehensive summary of existing projects investigating accessible gestural interaction. We also highlight the limits of current approaches and propose future working directions. Then, we present the design of an interactive map prototype that includes both a raised-line map overlay and gestural interaction for accessing different types of information (e.g., opening hours, distances). Preliminary results of our project show that basic gestural interaction techniques can be successfully used in interactive maps for visually impaired people.

Keywords: gestural interaction, accessibility, visual impairment, interactive maps, non-visual interaction
\end{abstract}

\section{Introduction}

The recent rise of multi-touch devices, has led to an increased use of those devices in daily life [1]. Yet, touch displays are poorly accessible without vision [2]. First, there is no cutaneous feedback. On touchscreens you cannot feel your way to an interactive component, whereas this is possible with a traditional button based interface. Second, it is hard to provide instructions for the next action to take (i.e. where to press on the screen). Third, in many applications the position of interactive elements on the screen is not fixed, while on button-based interfaces users can memorize the position of buttons [3]. Furthermore, visually impaired people fear accidentally activating features on touch screens [3]. Consequently, making multi-touch more accessible to visually impaired people is an important task. Kane, Morris, et al. [4] classified touch screen accessibility in three categories. First, "hardware-only" approaches apply hardware modifications on the touchscreen, such as gluing tactile dots and braille labels on the screen [5]. Second, "hybrid approaches" combine the use of hardware modifications, such as raised-line overlays, and audio output. Audio-tactile maps (interactive maps based on tactile maps and speech output [6]) are included in this 
category. Third, "software only" approaches make use of accessible gestural interaction combined with audio feedback. Indeed, gestural interaction might be a cheap and flexible way to improve accessibility of multi-touch technology. Yet, it has so far rarely been used in devices for visually impaired people and thus there is need for further investigations. We suggest that gestural interaction can augment "hybrid approaches", i.e. to combine gestural interaction with a raised-line overlay.

In this paper we focus on accessible gestural interaction. To our knowledge there is no comprehensive overview of existing research projects on accessible gestural interaction. Therefore, in the first section we investigate the current state of the art. We highlight existing solutions as well as their limits. Then, we present the design and preliminary evaluation of an accessible interactive map including both a raised-line map overlay and gestural interaction techniques. We conclude by opening up future research steps.

\section{State of the Art on Accessible Gestural Interaction}

Gestures are an integral part of human communication. In the context of HumanComputer Interaction, no standard definition exists but different authors have defined gestural interaction $[7,8]$. The term "gesture" defines dynamic and intentional movements of certain body parts (likely the hands and arms). Because these movements follow a defined path they can be recognized by a computer and trigger a command.

Some research projects have developed accessible gestural interaction. Slide Rule [3] provided a set of accessible gestures for touch screen interfaces. Basic interaction techniques have been developed, as for instance a one-finger scan for browsing lists, a second finger to tap and select items, a multi-directional flick for additional actions and a L-shaped gesture for browsing hierarchical information. Slide Rule proved to be significantly faster than the use of a Pocket PC device, even if users made more errors and results concerning satisfaction were contradictory. Wolf, Dicke, and Grasset [9] studied gestural interaction for spatial auditory interfaces on smartphones without specifically targeting visually impaired people. The study included not only $2 \mathrm{D}$ but also 3D gestures measured by the embedded smartphone sensors (e.g. accelerometer or gyroscope). Participants mostly created gestures that were executed on the touch surface, some gestures that were based on 3D movements of the phone and only few gestures that combined both. To our knowledge, no project has studied the use of 3D gestures for visually impaired people even though it would be interesting. NavTouch [10] provided a text-entry method for blind users on a multi-touch device. The alphabet was navigated by performing directional gestures on the screen, while constant audio feedback was given. Other projects aimed at including gestural interaction in interactive maps. Zeng and Weber [11] implemented basic gestures such as panning and zooming. Yatani et al. [12] proposed the use of flick gestures for navigating lists or selecting items. Carroll et al. [13] suggested using common gestures but replacing the action that is associated in visual interfaces with these gestures. Performing a twofinger pinch would then not result in changing the zoom level of the map, but in changing the type of displayed content. Finally, commercial smartphones start to pro- 
vide accessible gestural interaction. The most advanced accessible gestural interaction is provided by Apple with the VoiceOver Screen Reader [14].

\subsection{Design Guidelines for Accessible Gestural Interaction}

Only few guidelines for designing accessible gestural interaction exist. The Slide Rules project [3] led to the following design principles: prevent the risk of accidentally executing an action; provide a resolution adapted for the finger and not for the eye; reduce selection accuracy; provide quick browsing and navigation; make gestural mapping intuitive and enable a "return home" function. McGookin et al. [15] suggested not to use short impact related gestures-simple taps-as this led to unintended touch events; to avoid localized gestures or to provide a reference system; and to provide feedback for all actions. Kane, Wobbrock, and Ladner [16] proposed several guidelines for creating accessible gestures: avoid symbols and letters from print writing; position gestures in edges and on corners as cues for identifying one's position on the screen; expect low location accuracy; increase the time for executing gestures and use familiar layouts such as QWERTY keyboards.

\subsection{Challenges of Accessible Gestural Interaction}

As stated above, visually impaired users fear accidentally activating features by taping on the multi-touch screen [3]. Observations on unintended touch interaction of visually impaired participants have been made in different projects. El-Glaly, Quek, Smith-Jackson, \& Dhillon [17] proposed an application for allowing blind people to read books on an iPad. They observed unintended touch input from the palms resting on the touch surface, but also from other fingers unconsciously touching the surface while holding the device. The intended exploratory movement was a single finger moving over the surface in order to read the book. Because this movement was regular, they were able to track it and eliminate the unintended input. Furthermore, short impact related gestures, such as single taps, should be avoided because they are likely to occur accidentally $[12,15]$. As described above, placing interactive zones in the edges or corners of a device, might reduce the likelihood to trigger this interaction accidentally [16]. Despite these first recommendations and solutions, it remains a challenge for the research domain of multi-touch to find adapted interaction techniques for visually impaired people that avoid accidental touch interaction. Furthermore it can be observed that so far very few studies put the focus on gestural interaction that is designed specifically for visually impaired people and evaluated in order to ensure usability. In the future, visually impaired people should thus be included throughout the design process from the creation of ideas to the evaluation.

\section{Gestural Interaction for an Accessible Geographic Map}

Traditionally, geographic maps for visually impaired people are hard-copy maps with raised lines. With the rise of new technologies, research projects have been de- 
voted to the design of interactive maps for visually impaired people. The design of these maps varied in different aspects, such as content, devices and interaction techniques [18]. Many accessible interactive maps are based on the use of touch screens ranging from smartphones to large touch tables. Despite gestural interaction being usable by blind people [16], few of these projects made use of more complex gestures than tapping (see above). The information that accessible interactive maps provide is therefore often limited to basic information, such as street names [6].

Gestural interaction would provide the possibility to enrich interactive maps. First, gestures would enable the possibility to access important information, such as distances, directions, or itineraries. In the Talking TMAP prototype [19], which was based on a mono-touch display, the calculation of distances was executed with a combination of taps with a single finger. We suggest that using more than one finger could facilitate distance calculation. Second, it would be possible to present more complex information than names. Indeed, it is interesting for the user to choose the amount and type of information that is presented on the map and this possibility has been provided in prior maps. For instance, in the Talking TMAP project [19], different levels of information could be accessed through repeated tapping. This information included names of streets, spelling of the street name, address ranges and length of the street. Levesque et al. [20] compared three conditions for exploration of a tactile drawing by visually impaired people: 1 ) static content, 2) users could manually adapt the level of detail, 3) the level of detail was automatically determined from speed of exploratory movements. Although there was no significant difference in reading speed or error rate, users clearly preferred the mode in which they could manually toggle the amount of detail.

Taken together, this suggests the interest of providing access to more detailed information and of letting the user choose the level of detail that is displayed. Consequently, the aim of our project was to study how basic gestural interaction could be used to enrich a previously developed interactive map prototype (based on a multitouch device with raised-line overlay) [6] with extended functionality, such as adding supplementary audio output about opening hours, directions, etc.

\subsection{Designing the Prototype}

For the design of our interactive map prototypes we made use of participatory design methods and included visually impaired people throughout the design cycle [21]. Concretely for the design of gestural interaction, we conducted brainstorming sessions. The sessions took place between one blind expert and four sighted researchers. During the brainstorming sessions, we discussed how to make use of gestural interaction within the previously developed interactive map concept, i.e., touchscreen with raised-line overlay and audio output. We kept the idea of selecting different levels of information, as has been suggested in the literature. For instance with the same interactive map, it could be possible to switch between the audio output for basic points of interest, opening hours, or public transportation. We proposed the combined use of buttons and basic gestural interaction to access these different information levels, so that users could choose the content they were interested in. Also, we decided to ex- 
plore how distance information could be provided, because users in our previous studies had stated that distance information was important.

\subsection{Implementation of the Prototype}

Our interactive map prototype consisted of a raised-line map overlay placed over a multi-touch screen, a computer connected to the screen and audio output [6].

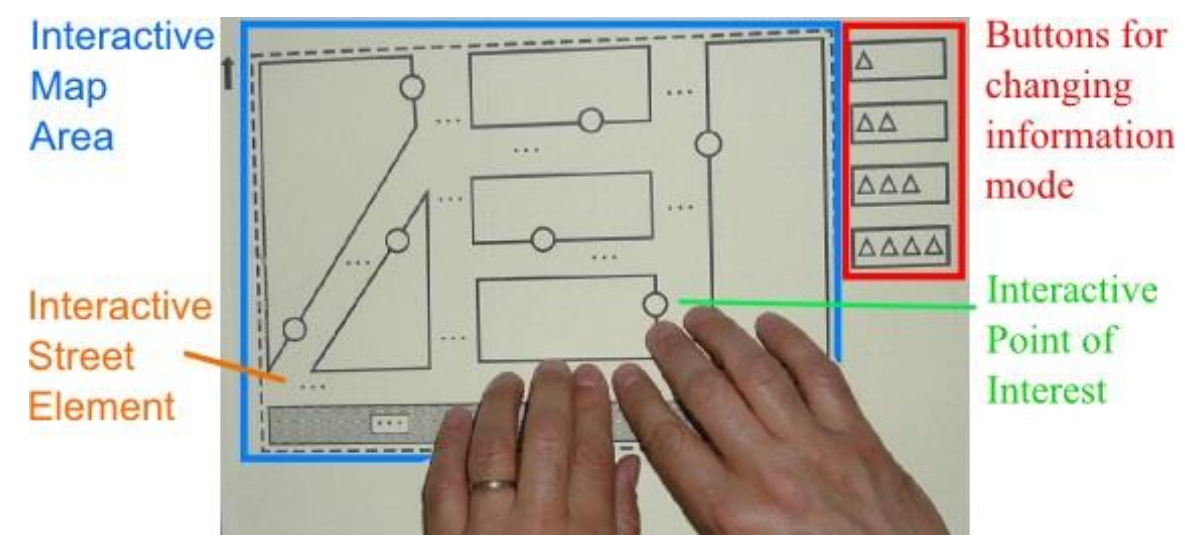

Fig. 1. The raised-line map drawing which is placed as an overlay on a multi-touch device.

The raised-line overlay (see Fig. 1) was based on the map drawing from previous studies [6] and included the geographical map (blue area in Fig. 1) as well as the button area (red zone in Fig. 1). We designed the map with Inkscape ${ }^{1}$ in $\mathrm{SVG}_{\text {format. }}$ SVG provides a text file that can be analyzed by an application as well as a visual representation. The visual view could then be printed as raised-line map. The SVG file was used by our application for extracting information on position and dimension of map elements. We came up with the idea of an additional configuration file that provided supplementary information on the map elements, such as opening hours, entry fees, length of a street, etc. This configuration file was written in XML. It was parsed by the application and then associated with the components in the SVG file.

\section{Choice of Interaction Techniques}

As stated before, the objective of this project was to test whether gestural interaction techniques were usable in interactive maps for visually impaired people. Interaction techniques comprised gestural input as well as audio output. For the latter we used the S.I. VOX / Vocalyze software ${ }^{2}$. We implemented gestural interaction with the MT4J Gestural API [22], an open-source and cross-platform framework for developing multi-touch applications. Names of elements were announced when double

1 http://inkscape.org/en [last accessed April 30 $\left.{ }^{\text {th }} 2014\right]$

2 http://users.polytech.unice.fr/ helen/SERVER_SI_VOX/pages/index.php?page=Accueil [last accessed April 30 ${ }^{\text {th }}$ 2014] 
tapping on interactive elements. Furthermore, we used basic gestural interaction techniques provided by the MT4J API. Among these, MT4J provided a lasso gesture, which means circling around a map element without lifting the finger (Fig. 2). Additionally, we implemented a tap and hold gesture. The user had to tap on a map element and maintain the finger pressed. A beep sound confirmed the activation. The user could then tap on a second map element and a second beep would confirm the activation (Fig. 2). The distance between both elements was then verbally announced.

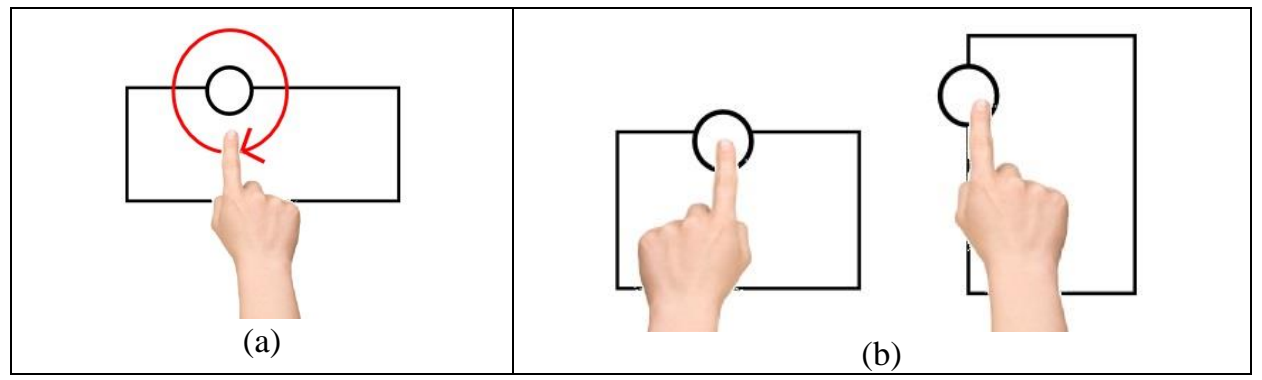

Fig. 2. Schema of the different gestural interaction techniques. (a) Lasso; (b) Tap and Hold

As previously discussed, visually impaired users want to choose the type of information ([19]). We decided to make this information accessible via modes, i.e. different system states. For changing modes, we added buttons next to the drawing of the geographic map. Buttons were ordered from level one to four by an increasing number of triangles (see red marking in Fig. 1). Double tapping on the button changed the mode and the name of the new mode was verbally announced. Each mode then provided access to different types of information via different interaction techniques (see Table 1). The goal was that users could actively change the kind of information presented on the map in accordance with the study by Levesque et al. [20].

\begin{tabular}{c|c|c|c|c}
\hline Mode & 1 & 2 & 3 & 4 \\
\hline \hline & & Double Tap & Double Tap & \\
Active Gestures & Double Tap & Lasso & Tap And Hold & Double Tap \\
\hline & & Street Names & Street Names & \\
& Street Names & Names of POI & Names of POI & Information on \\
Type of Information & Names of POI & Detailed Information POI & Distances between POI & Public Transport \\
\hline
\end{tabular}

Table 1. Distribution of gestures and types of information in the current implementation.

\subsection{Evaluation}

The present prototype is a proof-of-concept and currently only pretests have been done. The tests have been conducted with one blind researcher to check the functioning of different map features. This person had also tested the previous map prototype [6] and was thus familiar with the interactive map concept. The preliminary study showed that audio output was comprehensible, even if the voice was not perceived as 
pleasant. We detected that the user needed time to familiarize with the new gestures, but he was then able to use them for accessing different levels of information. The lasso gesture seemed to be challenging as it demanded to first identify a map element by following the raised lines and then circle around it. This is indeed more difficult without visual cues. There were no problems with accessing the different information levels by using the different buttons. Also the tap-and-hold gesture for the distance was usable and much appreciated.

\section{Discussion and Conclusion}

In this paper, we presented a state of the art of non-visual gestural interaction. Analysis of the literature shows different approaches to make gestural interaction accessible. It also reveals challenges that need to be addressed in the future, such as unintended touch input. Furthermore, we showed an example of how gestural interaction techniques can be used in interactive maps for visually impaired people. We checked that it was possible for a blind user to access distances and different types of information. Furthermore, our observations suggest that gestural interaction should be picked carefully. Gestures that are easy for sighted people may be less evident for visually impaired people (e.g., the lasso). This is in line with previous studies on accessible gestural interaction [16]. The present work only presented a proof-ofconcept. A more advanced design process would be necessary, as well as extended evaluations with several visually impaired users. In the future, it would be interesting to go beyond the basic gestural interaction provided by the API, and to design specific gestural interaction. To sum up, we believe that thoroughly designed gestural interaction would open up new interaction possibilities in research and commercial prototype. However, it remains a challenge for the research domain of multi-touch to find interaction techniques that are usable without sight. We suggest that future studies should address the design of specific gestures for visually impaired people by including them throughout the design process from the creation of ideas to the evaluation.

Acknowledgments. We thank Alexis Paoleschi who developed the prototype presented in this paper and our blind participants.

\section{References}

1. Schöning, J.: Touch the future: The recent rise of multi-touch interaction. J. Eur. Comm. Futur. Emerg. Technol. Proactive Initiat. Pervasive Adapt. 5531, 1-2 (2010).

2. Buxton, W.: Multi-Touch Systems that I Have Known and Loved. (2007).

3. Kane, S.K., Bigham, J.P., Wobbrock, J.O.: Slide Rule: making mobile touch screens accessible to blind people using multi-touch interaction techniques. ASSETS '08. pp. 73 80. ACM Press, New York, New York, USA (2008).

4. Kane, S.K., Morris, M.R., Wobbrock, J.O.: Touchplates: Low-Cost Tactile Overlays for Visually Impaired Touch Screen Users. ASSETS'13. ACM, Bellevue, Washington, USA (2013). 
5. Xu, S., Bailey, K.: Design Touch Feedback for Blind Users. In: Stephanidis, C. (ed.) HCI International 2013 Volume 373. pp. 281-285. Springer Berlin Heidelberg, Berlin, Heidelberg (2013).

6. Brock, A.M., Truillet, P., Oriola, B., Picard, D., Jouffrais, C.: Design and User Satisfaction of Interactive Maps for Visually Impaired People. In: Miesenberger, K., Karshmer, A., Penaz, P., and Zagler, W. (eds.) ICCHP 2012. LNCS, vol. 7383. pp. 544-551. Springer, Linz, Austria (2012).

7. Pavlovic, V.I., Sharma, R., Huang, T.S.: Visual interpretation of hand gestures for human-computer interaction: a review. IEEE Trans. Pattern Anal. Mach. Intell. 19, 677-695 (1997).

8. Kamber, Y.: Empirical Investigation of The Memorability of Gesture Sets, (2011).

9. Wolf, K., Dicke, C., Grasset, R.: Touching the void: gestures for auditory interfaces. TEI '11. pp. 305-308. ACM Press, New York, New York, USA (2011).

10. Guerreiro, T., Lagoá, P., Nicolau, H., Gonçalves, D., Jorge, J.A.: From Tapping to Touching: Making Touch Screens Accessible to Blind Users. IEEE Multimed. 15, 48-50 (2008).

11. Zeng, L., Weber, G.: Audio-Haptic Browser for a Geographical Information System. In: Miesenberger, K., Klaus, J., Zagler, W., and Karshmer, A. (eds.) ICCHP 2010. LNCS, vol. 6180. pp. 466-473. Springer, Heidelberg (2010).

12. Yatani, K., Banovic, N., Truong, K.: SpaceSense: representing geographical information to visually impaired people using spatial tactile feedback. CHI '12. pp. 415 424. ACM Press, New York, New York, USA (2012).

13. Carroll, D., Chakraborty, S., Lazar, J.: Designing Accessible Visualizations: The Case of Designing a Weather Map for Blind Users. Universal Access in Human-Computer Interaction. Design Methods, Tools, and Interaction Techniques for eInclusion. pp. 436-445. Springer Berlin Heidelberg (2013).

14. Apple: Apple - Accessibility - OS X - VoiceOver, http://www.apple.com/accessibility/osx/voiceover/.

15. McGookin, D., Brewster, S.A., Jiang, W.W.: Investigating touchscreen accessibility for people with visual impairments. NordiCHI. pp. 298-307. ACM (2008).

16. Kane, S.K., Wobbrock, J.O., Ladner, R.E.: Usable gestures for blind people. CHI '11. pp. 413-422. ACM Press, Vancouver, BC, Canada (2011).

17. El-Glaly, Y., Quek, F., Smith-Jackson, T., Dhillon, G.: Audible rendering of text documents controlled by multi-touch interaction. ICMI'12. pp. 401-408. ACM Press, New York, New York, USA (2012).

18. Brock, A.M., Oriola, B., Truillet, P., Jouffrais, C., Picard, D.: Map design for visually impaired people: past, present, and future research. Médiation Inf. - Handicap Commun. 36, 117-129 (2013).

19. Miele, J. a., Landau, S., Gilden, D.: Talking TMAP: Automated generation of audiotactile maps using Smith-Kettlewell's TMAP software. Br. J. Vis. Impair. 24, 93-100 (2006).

20. Lévesque, V., Petit, G., Dufresne, A., Hayward, V.: Adaptive level of detail in dynamic, refreshable tactile graphics. IEEE Haptics Symp. 1-5 (2012).

21. Brock, A.M., Vinot, J.-L., Oriola, B., Kammoun, S., Truillet, P., Jouffrais, C.: Méthodes et outils de conception participative avec des utilisateurs non-voyants. IHM'10 pp. 65 - 72. ACM Press, Luxembourg, NY, USA (2010).

22. Laufs, U., Ruff, C., Weisbecker, A.: MT4j - an open source platform for multi-touch software development. VIMation J. 58 - 64 (2010). 